\title{
In vitro and in vivo activity of GT-1, a novel siderophore cephalosporin, and GT-055, a broad-spectrum $\beta$-lactamase inhibitor, against biothreat and ESKAPE pathogens
}

\author{
Stephanie A. Halasohoris ${ }^{1}$ - Jennifer M. Scarff ${ }^{1}$ 'Lisa M. Pysz ${ }^{1}$ - Sanae Lembirik ${ }^{1}$ Margaret M. Lemmon ${ }^{1}$. \\ Donald Biek $^{2} \cdot$ Brendan Hannah $^{2} \cdot$ Steven D. Zumbrun ${ }^{1} \cdot$ Rekha G. Panchal $^{1}$
}

Received: 15 June 2021 / Revised: 29 July 2021 / Accepted: 12 August 2021 / Published online: 14 September 2021

This is a U.S. government work and not under copyright protection in the U.S.; foreign copyright protection may apply 2021

\begin{abstract}
Antimicrobial-resistance (AMR) has become an increasingly difficult issue to overcome for bacteria associated with both community- and hospital-acquired infections as well as potential biodefense threats. The need to identify new therapeutics of novel classes and/or with unique mechanisms is critical to combatting AMR in the coming years. GT-1 (LCB10-0200), a siderophore-linked cephalosporin, is one such novel option and is formulated to be used either alone or in combination with a novel broad-spectrum $\beta$-lactamase inhibitor, GT-055 (LCB18-055). This study assessed the in vitro and in vivo efficacy of GT-1 and GT-055 against a broad array of multi-drug resistant and biothreat pathogens. Here, we demonstrated sub- $4 \mu \mathrm{g} \mathrm{ml}{ }^{-1}$ efficacy against a number of pathogens in vitro. We further determined that in mice infected via aerosol route with Yersinia pestis, efficacy of GT-1/GT-055 treatment is at least equivalent to the comparator antibiotic, ciprofloxacin.
\end{abstract}

\section{Introduction}

The CDC estimates that there are over 2.8 million infections that result in almost 36,000 deaths from drug-resistant bacteria and fungi per year [1]. It has been over a decade since attention was brought to the need for new therapeutics against the difficult-to-treat ESKAPE (Enterococcus faecium, Staphylococcus aureus, Klebsiella pneumoniae, Acinetobacter baumannii, Pseudomonas aeruginosa, and Enterobacter species) pathogens [2, 3]. And yet, in a 2019 CDC report, multiple drug-resistant (MDR) infections increased in frequency from 2012 to 2017, including extended spectrum $\beta$-lactamase (ESBL) producing Enterobacterales (e.g., $K$. pneumoniae and E. coli). The CDC ranks drugresistant bacteria as urgent, serious, or concerning threats and

Supplementary information The online version contains supplementary material available at https://doi.org/10.1038/s41429021-00472-9.

Rekha G. Panchal

rekha.g.panchal.civ@mail.mil

1 US Army Medical Research Institute of Infectious Diseases, Fort Detrick, MD, USA

2 Geom Therapeutics Inc, San Diego, CA, USA four $\beta$-lactam antibiotic-resistant pathogens are on these lists. Carbapenem-resistant Enterobacterales and carbapenemresistant Acinetobacter were ranked as urgent threats while multidrug-resistant $P$. aeruginosa and ESBL-producing Enterobacterales were ranked as serious threats [1]. This highlights the need for new antibiotics or combinations that circumvent these resistance mechanisms.

In addition to the ESKAPE pathogens, infections by biodefense pathogens would also benefit from improved treatments. Burkholderia pseudomallei infections are particularly difficult to treat, with a months-long treatment regimen for which failures are not uncommon. B. pseudomallei has a reduced membrane permeability compared to other Gramnegative bacteria. The resistance to $\beta$-lactam antibiotics can be mediated through the PenA $\beta$-lactamase or through mutations and gene rearrangements [4]. These acquired resistances have a low frequency of occurrence, but given the low number of available antibiotics to treat $B$. pseudomallei infections, they remain an issue of concern. Infections by another biodefense pathogen, Yersinia pestis have a small window for initiation of effective treatment and there exist only a few recommended antibiotics [5].

GT-1 (LCB10-0200) is a novel therapeutic that consists of a siderophore, dihydroxypyridone, conjugated to a modified aminothiazoylglycyl cephalosporin [6]. The goal of this approach is to exploit the bacterial siderophore uptake 
systems to enable antibiotic entry in a "Trojan horse" strategy, similar to the strategy successfully employed by cefiderocol [7]. In addition, the conjugated molecule is resistant to hydrolysis by many ESBLs and carbapenemases. GT-1 was more effective against clinical isolates of $P$. aeruginosa, Klebsiella oxytoca, Proteus spp., Serratia marcescens, and Enterobacter aerogenes than ceftazidime and ceftriaxone. This improved activity was also observed against $\beta$-lactamaseproducing strains of $P$. aeruginosa [6]. GT-1 also resulted in a larger reduction in bacterial load than ceftazidime when used to treat four strains of $P$. aeruginosa in murine systemic and thigh infection models [6].

$\beta$-lactam antibiotics are often coupled with a $\beta$-lactamase inhibitor as a way to counteract the enzymes produced by resistant bacteria. To that effect, the efficacy of GT- 1 when coupled with GT-055 (LCB18-055), a novel $\beta$-lactam inhibitor (BLI), was also investigated [8]. The chemical structures of both GT-1 and GT-055 are shown in Fig. 1. The GT-1/GT-055 combination was tested against $E$. coli, $K$. pneumoniae, and Acinetobacter spp. isolates with a variety of $\beta$-lactamase profiles. Against all three bacterial genera, GT-1 alone had lower MICs against most strains than ceftazidime alone. GT-1/GT-055 in combination had reduced MICs against many strains compared to GT-1 alone and was better than the combination of ceftazidime/avibactam, particularly against $E$. coli and $K$. pneumoniae. It has been found that cefiderocol is active in the presence of many $\beta$-lactam hydrolyzing enzymes, even without the addition of a $\beta$-lactamase inhibitor $[9,10]$.

Pharmacokinetic (PK) studies of GT-1 in mouse, rat and dog have been reported. The PK parameters of GT-1 across different species suggest low clearance, low volume of distribution, and a short half-life, with a high free drug fraction

\section{GT-1: Siderophore Cephalosporin}
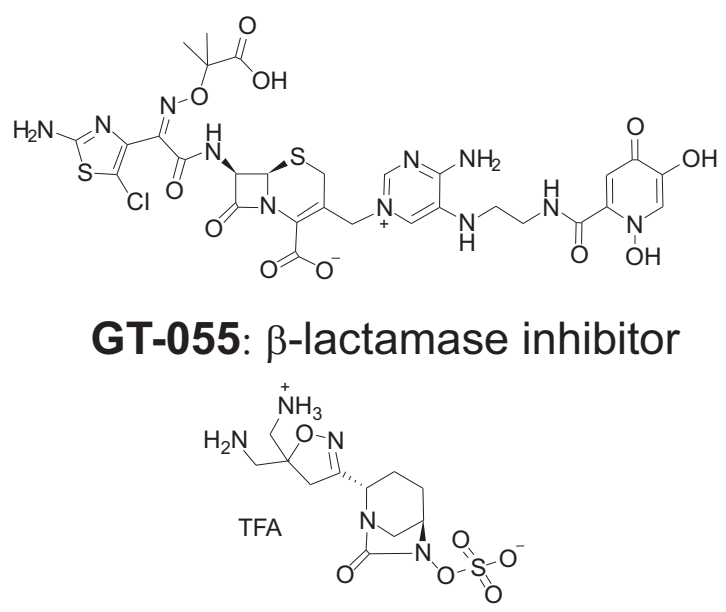

Fig. 1 Chemical structures of GT-1 (LCB10-0200) and GT-055 (LCB18-055)
[11]. Dose-fractionation studies demonstrated that the PK/ Pharmacodynamic (PD) index associated with GT-1 efficacy in the mouse thigh model is plasma free-drug $\% \mathrm{~T}>\mathrm{MIC}$. The free-drug \% $>$ MIC associated with net bacterial stasis, 1-log kill, and 2-log kill are 40.2, 58.3, and 85.8, respectively for Enterobacterales and P. aeruginosa [12]. Studies of GT-1 efficacy in a mouse lung infection model have also been performed and PK/PD targets determined [13]. The PK characteristics of GT-055 are similar to those of GT-1, and dose-fractionation studies demonstrated that the PK/PD index associated with GT-055 efficacy in a one-compartment in vitro infection model is plasma free-drug AUC/MIC ratio. For Enterobacterales, the free-drug AUC/MIC ratios associated with net bacterial stasis, 1-log kill, and 2-log kill are 4.13, 13.7, 57.2, respectively [14]. Initial PK/PD studies found the optimal GT-1:GT-055 ratio to be 1:1.

Here, we further characterize the activity of GT-1 and GT-055 against MDR bacteria, particularly those with carbapenem and other $\beta$-lactam resistances. We investigate the in vitro efficacy of these compounds against the biodefense pathogens Bacillus anthracis, Burkholderia mallei, B. pseudomallei, Francisella tularensis, and Y. pestis. GT-1 and GT-1/GT-055 were tested in mouse infection models of B. pseudomallei and $Y$. pestis. The combination had similar efficacy as the therapeutic standard, ciprofloxacin, in the $Y$. pestis model, although it was less active in the B. pseudomallei infection model.

\section{Materials and methods}

\section{Compounds and bacterial strains}

GT-1 and GT- 055 were provided by Legochem Biosciences (Seoul, South Korea). The activity and structures have been previously published for both GT-1 [6] and GT-055 [8]. The AR Bank resistant panels used here, Enterobacterales Carbapenem Breakpoint, Gram-negative Carbapenemase Detection, EnterobacteralesCarbapenemase Diversity, and Isolates with New or Novel Antibiotic Resistance panels were obtained from the CDC (Atlanta, GA). The B. anthracis, $B$. mallei, B. pseudomallei, $F$. tularensis, and $Y$. pestis strains were obtained from the Unified Culture Collection at USAMRIID (Frederick, MD). The control strains for MIC determination, S. aureus (ATCC 29213), E. coli (ATCC 25922), and $P$. aeruginosa (ATCC 27853) were obtained from the ATCC (Manassas, VA).

\section{MIC determination for bacteria from CDC resistance panels}

The minimum inhibitory concentration (MIC) of GT-1 and GT-055 either alone or in combination was determined for 
bacteria in the following panels obtained from the Centers for Disease Control and Prevention (CDC, USA) and U.S. Food and Drug Administration (FDA) Antibiotic Resistance Isolate Bank: The entirety of the Enterobacterales Carbapenem Breakpoint, Gram-negative Carbapenemase Detection, and Enterobacterales Carbapenemase Diversity panels, and select isolates from the collection of New or Novel Antibiotic Resistance panel. The $\beta$-lactamase resistance genes in the strains of these panels was obtained from the CDC AR Bank [15]. The control strains for the assays were obtained from the ATCC: Escherichia coli (ATCC 25922), Staphylococcus aureus (ATCC 29213), and Pseudomonas aeruginosa (ATCC 27853).

The MICs were determined in accordance with CLSI guidelines [16, 17]. The CDC resistance panel strains were grown on Mueller Hinton agar overnight at $37^{\circ} \mathrm{C}$. Bacterial colonies were transferred into PBS with a cotton swab and the $\mathrm{OD}_{600}$ of the suspension was determined. The amount of bacteria to add to the MIC inoculum was calculated based on the approximated $\mathrm{CFU} \mathrm{ml}{ }^{-1}$ of an $\mathrm{OD}_{600}=1$ for each strain and the final concentration of bacteria in the assay, $5 \times 10^{5}$ $\mathrm{CFU} \mathrm{ml}{ }^{-1}$. The bacteria were diluted into cation-adjusted Mueller Hinton broth (CAMHB) and added to the 96-well plates that contained the serial dilutions of the compounds. Plates were incubated at $37^{\circ} \mathrm{C}$ with $5 \% \mathrm{CO}_{2}$ for $16-20 \mathrm{~h}$. The MIC was determined as the lowest concentration of compound that inhibited growth of the bacteria.

\section{MIC determination in iron-depleted media}

The MICs for the compounds against bacteria grown in irondepleted CAMHB (ID-CAMHB) were compared to the MICs determined in CAMHB for certain strains. To deplete the iron, $150 \mathrm{~g}$ Chelex (Bio-Rad Laboratories) was added per $150 \mathrm{ml}$ CAMHB. After $2 \mathrm{~h}$ at room temperature, the medium was filtered with a $0.2 \mu \mathrm{m}$ filter. The cation levels were adjusted such that the final concentrations were: $\mathrm{Ca}^{2+}$ $\left(20-25 \mathrm{mg} \mathrm{l}^{-1}\right) ; \mathrm{Mg}^{2+}\left(10-12.5 \mathrm{mg} \mathrm{l}^{-1}\right) ; \mathrm{Zn}^{2+}\left(10 \mu \mathrm{M}, \mathrm{ZnSO}_{4}\right)$ and the $\mathrm{pH}$ was adjusted to 7.2-7.4, if necessary. The medium was filtered with a $0.2 \mu \mathrm{m}$ filter again after these adjustments to ensure sterility.

B. mallei and B. pseudomalle $i$ were grown on chocolate agar at $35^{\circ} \mathrm{C}$ for $24 \mathrm{~h}, F$. tularensis was grown on chocolate agar at $35^{\circ} \mathrm{C}$ for $48 \mathrm{~h}$, and $Y$. pestis was grown on sheep blood agar at $28{ }^{\circ} \mathrm{C}$ for $24 \mathrm{~h}$. Other strains tested, including the QC strains (E. coli ATCC 25922, S. aureus ATCC 29213, P. aeruginosa ATCC 27853), were grown on sheep blood agar at $35^{\circ} \mathrm{C}$ for $24 \mathrm{~h}$. Bacteria were resuspended to 0.5 McFarland and diluted 1:100 in media (CAMHB or IDCAMHB). The medium for $F$. tularensis was supplemented with $2 \%$ IsoVitalex (BD). A volume of $50 \mu \mathrm{l}$ of the diluted bacteria were added to the microtiter plates for a final concentration of $5 \times 10^{5} \mathrm{CFU} \mathrm{ml}^{-1}$ in $100 \mu \mathrm{l}$. The plates were incubated at $35^{\circ} \mathrm{C}$ for $18-24 \mathrm{~h}$ ( $48 \mathrm{~h}$ for $F$. tularensis) and the MICs were recorded. Representative strains were used for initial susceptibility experiments and then 30 strains of $B$. pseudomallei, B. mallei, and $Y$. pestis were used for determination of $\mathrm{MIC}_{90}$ s.

\section{In vivo efficacy}

Animal research at the United States Army Medical Research Institute of Infectious Diseases (USAMRIID) was conducted under an animal use protocol approved by the USAMRIID Institutional Animal Care and Use Committee in compliance with the Animal Welfare Act, PHS Policy, and other Federal statutes and regulations relating to animals and experiments involving animals. The facility is accredited by the Association for Assessment and Accreditation of Laboratory Animal Care International and adheres to principles stated in the Guide for the Care and Use of Laboratory Animals (National Research Council, 2011).

Six- to eight-week old BALB/c mice were challenged with B. pseudomallei strain $1026 \mathrm{~b}$ via whole body aerosol. It is possible that re-infection could potentially occur by mice licking their fur, although we have no evidence for this. The aerosol was generated with a three-jet collison nebulizer [18]. All aerosol procedures were controlled and monitored by the Automated Bioaerosol Exposure system [19] operating with a whole-body rodent exposure chamber. Integrated air samples were collected from the chamber during each exposure into an all-glass impinger (AGI). A sample from the AGI was serially diluted and plated on SBA to determine the concentration of bacteria. The challenge dose (CFU per mouse) of B. pseudomallei which corresponds to the dose introduced in the chamber was estimated using Guyton's formula [20]. Since not all mice could be aerosol challenged in a single spray, mice from each run were randomized into the treatment groups ( $n=10$ mice) such that each group contained a similar number of mice from each spray. The mean deposited dose for B. pseudomallei was $4.09 \times \mathrm{LD}_{50} \mathrm{~s}$ (3 separate sprays of $5.56,3.49$, and $\left.3.22 \times \mathrm{LD}_{50} \mathrm{~s}\right)$. Treatment was initiated at $12 \mathrm{~h}$ post-infection and $0.2 \mathrm{ml}$ was administered via either intraperitoneal (IP) or subcutaneous (SC) injection. The treatment groups were: saline (IP); ceftazidime $\left(300 \mathrm{mg} \mathrm{kg}^{-1}\right.$; IP); one of three doses of GT-1 $\left(30,60\right.$, or $120 \mathrm{mg} \mathrm{kg}^{-1}$; SC); or one of three doses of GT-1/GT-055 at 1:1 ratio $(30 / 30,60 / 60$, or $120 / 120 \mathrm{mg} \mathrm{kg}^{-1}$; SC). Two additional groups of mice had treatment initiated at $6 \mathrm{~h}$ post-infection: GT-1 $\left(120 \mathrm{mg} \mathrm{kg}^{-1}\right.$, SC) and GT-1/GT-055 1:1 ratio (120/120 $\mathrm{mg} \mathrm{kg}^{-1}$; SC). Treatment was $\mathrm{q} 6 \mathrm{~h}$ and continued for 21 days. Mice were closely monitored for morbidity and moribund mice were euthanized. At day 55 post-infection, the surviving mice were euthanized and necropsies completed to harvest the spleens. The spleens were homogenized and serially diluted for enumeration of the bacterial load. 
Table 1 Summary of MIC to GT-1/GT-055

\begin{tabular}{lllll}
\hline \multirow{2}{*}{ Bacteria } & \multicolumn{4}{l}{ Number of strains with MIC } \\
\cline { 2 - 5 } & $\leq 4 / 4$ & $8 / 8-32 / 32$ & $>32 / 32$ & Total \\
& $\mu \mathrm{g} / \mathrm{ml}$ & $\mu \mathrm{g} / \mathrm{ml}$ & $\mu \mathrm{g} / \mathrm{ml}$ & \\
\hline Acinetobacter baumannii & 4 & 4 & 6 & 14 \\
Citrobacter species & 5 & 1 & 1 & 7 \\
Enterobacter aerogenes & 4 & 2 & 0 & 6 \\
Enterobacter cloacae & 14 & 2 & 0 & 16 \\
Escherichia coli & 28 & 3 & 3 & 34 \\
Klebsiella spp. & 5 & 0 & 0 & 5 \\
(not pneumoniae) & & & & \\
Klebsiella pneumoniae & 34 & 3 & 14 & 51 \\
Kluyvera ascorbate & 1 & 0 & 0 & 1 \\
Morganella morganii & 0 & 1 & 1 & 2 \\
Proteus mirabilis & 2 & 1 & 2 & 5 \\
Providencia spp. & 1 & 1 & 0 & 2 \\
Pseudomonas aeruginosa & 6 & 3 & 3 & 12 \\
Raoultella ornithinolytica & 1 & 0 & 0 & 1 \\
Salmonella spp. & 1 & 1 & 0 & 2 \\
Serratia marcescens & 7 & 0 & 2 & 9 \\
Shigella sonnei & 1 & 0 & 0 & 1 \\
Total & $\mathbf{1 1 4}$ & $\mathbf{2 2}$ & $\mathbf{3 2}$ & $\mathbf{1 6 8}$ \\
\hline
\end{tabular}

Six- to eight-week old BALB/c were challenged with $Y$. pestis strain $\mathrm{CO} 92$ by intranasal inoculation. The mice were anesthetized with $0.1 \mathrm{ml}$ ketamine-acetylpromazine-xylazine (6.7 $\mathrm{mg} \mathrm{ml}^{-1}, 0.1 \mathrm{mg} \mathrm{ml}^{-1}, 0.7 \mathrm{mg} \mathrm{ml}^{-1}$, respectively) and 20 $\mu \mathrm{l}$ of bacteria were instilled into a single nare. The challenge dose was $2.9 \times 10^{4} \mathrm{CFU}\left(110 \mathrm{X} \mathrm{LD}_{50}\right)$ [21]. Treatment was initiated at $6 \mathrm{~h}$ post infection and consisted of the groups administered in $0.2 \mathrm{ml}$ : saline (SC; q6h); Ciprofloxacin (30 $\mathrm{mg} \mathrm{kg}^{-1}$; IP; q12h); one of three doses of GT-1 $(20,60$, or $200 \mathrm{mg} \mathrm{kg}^{-1}$; SC; q6h); or one of three doses of GT-1/GT055 at $2: 3$ ratio $\left(20 / 30,60 / 90\right.$, or $200 / 300 \mathrm{mg} \mathrm{kg}^{-1}$; SC; q6h) with 10 mice per group. Treatment continued for 7 days postinfection and mice were monitored for signs of morbidity for at least 30 days. Moribund mice were euthanized. After day 30 , up to 3 surviving mice per group were euthanized and necropsies completed to remove the lungs and spleens. The organs were homogenized in PBS and serially diluted for enumeration of bacterial load.

\section{Results}

\section{MIC of GT-1 and GT-055 against AMR bacteria}

GT-1 and GT-055 were tested alone and in combination (at 1:1 and 2:1 ratios, respectively) against four panels from the CDC and FDA Antibiotic Resistance Isolate Bank (Supplementary Table 1). Three of the panels were the
Enterobacterales Carbapenem Breakpoint, Gram-negative Carbapenemase Detection, and Enterobacterales Carbapenemase Diversity sets. Select strains from the Novel Antibiotic Resistance panel were also used for antimicrobial susceptibility testing. The majority of the strains were not sensitive to GT-055; only $28 / 168$ had an MIC that was $\leq 4 \mu \mathrm{g} \mathrm{ml}^{-1}$. Likewise, only $52 / 168$ strains had MICs $\leq 4 \mu \mathrm{g} \mathrm{ml}^{-1}$ for GT-1 alone. The combinations of GT- 1 and GT-055 had improved MICs compared to each compound alone. For the 1:1 ratio, $114 / 168$ strains had MICs $\leq 4 \mu \mathrm{g} \mathrm{ml}^{-1}$ (Table 1) and for the 2:1 ratio, 99/168 strains had MICs $\leq 4 \mu \mathrm{g} \mathrm{ml}^{-1}$ (value is for concentration of GT-1). GT-055 has intrinsic antibacterial activity against some isolates, and the increase in number of isolates inhibited at $\leq 4 \mu \mathrm{g} \mathrm{ml}^{-1}$ for the $1: 1$ ratio compared to 2:1 may reflect the combination of increased intrinsic activity at higher GT-055 concentrations and inhibition owing to inactivation of $\beta$-lactamases in the presence of GT-1.

We analyzed the strains that were not sensitive to $1: 1$ GT-1:GT-055 (MIC $\geq 8 \mu \mathrm{g} \mathrm{ml}^{-1}$ ) to determine if particular bacterial genera or the presence of resistance genes resulted in an increased likelihood of resistance. A. baumannii had the highest percentage of strains that were not sensitive, 10/14 strains (Table 1). Of the bacterial species for which there were more than ten strains in the panels, E. cloacae had 2/14, E. coli had 6/34, K. pneumoniae had 17/51, and $P$. aeruginosa had $6 / 12$ strains that were not sensitive to the 1:1 combination of GT-1 and GT-055 (Table 1). The antibiotic resistance genes that are present in the strains in the CDC AR bank panels are known and shared on the CDC AR Isolate Bank website [15]. Ten genes associated with $\beta$-lactamase resistance were present in more than 10 strains of bacteria, and only one was associated with resistance to the GT- 1 and GT- 055 combination treatment in a majority of the strains. NDM-1 was present in 28 strains, and 23 of those were resistant to GT-1/GT-055 combination (Supplemental Table 1). About half of strains with OXA-1 (16/31), OXA-50 (6/12), PAO (6/12), and SHV-1 (9/18) were also resistant to the combination. Fewer than $50 \%$ of the strains with the other genes, CTX-M-15 (16/38), KPC3 (1/20), OXA-9 (9/27), TEM-1A (7/28), TEM-1B (12/41) were resistant to the combination.

\section{MICs of GT-1 and GT-055 against biothreat bacteria}

The MICs of GT- 1 alone or in combination with GT-055 against biothreat bacteria were determined in both standard cation-adjusted Mueller Hinton broth (CAMHB) and irondepleted CAMHB (ID-CAMHB). When tested against representative strains, GT-1, GT-1:GT-055 (1:1), and GT-1 with GT-055 (fixed $4 \mu \mathrm{g} \mathrm{ml}^{-1}$ ) exhibited potent activity against B. pseudomallei, $Y$. pestis, and $F$. tularensis, were less active against $B$. malle $i$, and inactive against $B$. anthracis (Table 2). The MICs were lower for the bacteria grown in 
Table 2 MIC $^{\text {a }}$ against representative strains of biothreat bacterial pathogens

\begin{tabular}{lllllll}
\hline & Media & B. anthracis Ames & B. pseudomallei K96243 & B. mallei FMH & F. tularensis Schu4 & Y. pestis CO92 \\
\hline GT-1 & CAMHB & $>128$ & $\leq 0.03$ & 16 & 2 & 2 \\
& ID-CAMHB & $>128$ & 0.06 & 8 & 2 & 0.25 \\
GT-1/GT-055 (2:1) & CAMHB & $>128 / 64$ & $\leq 0.013 / 0.015$ & $16 / 8$ & 2 & $1 / 0.5$ \\
& ID-CAMHB & $>128 / 64$ & $0.125 / 0.06$ & $4 / 2$ & 4 & $0.25 / 0.125$ \\
GT-1 + GT-055 (4 $\mu \mathrm{g} / \mathrm{ml})$ & CAMHB & $>128 / 4$ & $\leq 0.03 / 4$ & $8 / 4$ & $4 / 4$ & $0.5 / 4$ \\
& ID-CAMHB & $>128 / 4$ & $0.125 / 4$ & $1 / 4$ & $4 / 4$ & $0.12 / 4$ \\
GT-055 & CAMHB & $>64$ & $>32$ & $>64$ & $>32$ & $>32$ \\
& ID-CAMHB & $>64$ & $>64$ & 64 & 32 & $>32$ \\
DOX & CAMHB & 0.06 & 1 & 0.25 & 0.25 & $\leq 0.015$ \\
\end{tabular}

${ }^{\mathrm{a}} \mathrm{MIC}(\mu \mathrm{g} / \mathrm{ml})$ of GT-1 or GT-055 or GT-1/GT-055 in combination

ID-CAMHB than those grown in CAMHB for B. mallei and $Y$. pestis and were similar between the conditions for $B$. pseudomallei and $F$. tularensis. The $\mathrm{MIC}_{90} \mathrm{~s}$ were calculated after the MIC was determined for 30 strains of B. mallei, B. pseudomallei, and $Y$. pestis (Table 3 ). The $\mathrm{MIC}_{90}$ values against these strains mirrored what was seen with the representative strains; the $\mathrm{MIC}_{90} \mathrm{~s}$ for $B$. mallei and $Y$. pestis were lower in the ID-CAMHB than they were in the CAMHB. In B. mallei, the $\mathrm{MIC}_{90} \mathrm{~s}$ (ID-CAMHB vs CAMHB) were 8 vs $32 \mu \mathrm{g} \mathrm{ml}^{-1}$ for GT-1, 4/2 vs $8 / 4 \mu \mathrm{g} \mathrm{ml}^{-1}$ for GT-1:GT-055 (2:1), and $2 / 4$ vs $8 / 4 \mu \mathrm{g} \mathrm{ml}^{-1}$ for GT-1 with a fixed $4 \mu \mathrm{g} \mathrm{ml}^{-1}$ concentration of GT-055. The $Y$. pestis $\mathrm{MIC}_{90} \mathrm{~s}$ (ID-CAMHB vs CAMHB) were 0.5 vs $2 \mu \mathrm{g} \mathrm{ml}^{-1}$ for GT-1, $0.5 / 0.25$ vs $2 / 1$ $\mu \mathrm{g} \mathrm{ml}^{-1}$ GT-1:GT-055 (2:1), and 0.12/4 vs $1 / 4 \mu \mathrm{g} \mathrm{ml}^{-1}$ for GT- 1 with a fixed $4 \mu \mathrm{g} \mathrm{ml}^{-1}$ concentration of GT-055. The $B$. pseudomallei $\mathrm{MIC}_{90} \mathrm{~S}$ were actually higher in the ID-CAMHB versus the CAMHB for GT-1 $\left(0.12\right.$ vs $\left.\leq 0.03 \mu \mathrm{g} \mathrm{ml}^{-1}\right)$, GT-1 at $2: 1$ ratio with GT-055 $(0.25 / 0.125$ vs $\leq 0.03 /$ $\leq 0.015 \mu \mathrm{g} \mathrm{ml}^{-1}$ ), or GT-1 with a fixed concentration of GT$055\left(0.06 / 4\right.$ vs $\left.\leq 0.03 / 4 \mu \mathrm{g} \mathrm{ml}^{-1}\right)$, but had low $\mathrm{MIC}_{90}$ s for both conditions.

\section{In vivo efficacy}

$\mathrm{BALB} / \mathrm{c}$ mice were challenged via aerosol with $4 \times \mathrm{LD}_{50}$ of B. pseudomallei strain $1026 \mathrm{~b}$ ( $\mathrm{MIC} \leq 0.03 \mu \mathrm{g} \mathrm{ml}^{-1}$ ). A 21 day treatment regimen of GT-1 alone or in combination with GT055 was initiated. Only one of the groups that received GT-1 alone or GT-1 and GT-055 (1:1 ratio) at $12 \mathrm{~h}$ post-infection had $10 \%$ survival, while the group that received $120 / 120 \mathrm{mg}$ $\mathrm{kg}^{-1}$ GT-1/GT-055 exhibited $30 \%$ survival (Table 4). Mice had a median survival of 7.5-8.5 days for the three doses of GT-1 tested, 30, 60, and $120 \mathrm{mg} \mathrm{kg}^{-1}$. When mice received GT-055 with GT-1, the median survival was higher than that observed for mice that only received GT-1 with the largest increase occurring in the highest dose group: 7.5 days (GT-1, $30 \mathrm{mg} \mathrm{kg}^{-1}$ ) vs 10.5 days (GT-1/GT-055, 30/30 $\mathrm{mg} \mathrm{kg}^{-1}$ );
8.5 days (GT-1, $60 \mathrm{mg} \mathrm{kg}^{-1}$ ) vs 14.5 days (GT-1/GT-055, 60/ $60 \mathrm{mg} \mathrm{kg}^{-1}$ ); and 8 days (GT-1, $120 \mathrm{mg} \mathrm{kg}^{-1}$ ) vs 42.5 days (GT-1/GT-055, 120/120 $\mathrm{mg} \mathrm{kg}^{-1}$ ). A similar increase in survival occurred when the highest dose was initiated at $6 \mathrm{~h}$ postinfection; the median survival of mice that received $120 \mathrm{mg} \mathrm{kg}^{-1}$ GT-1 or 120/120 $\mathrm{mg} \mathrm{kg}^{-1}$ GT-1/GT-055 were 12 and 42.5 days, respectively. The bacterial burden was then determined in the spleens of mice that survived in each group. Bacteria were not detected in the spleens of the mice that received the $120 / 120 \mathrm{mg} \mathrm{kg}^{-1}$ GT-1/GT-055 dose initiated at $12 \mathrm{~h}$ post-infection, whereas the other survivors had enlarged spleens and at least $10^{8} \mathrm{CFU} /$ spleen (Table 5). The bacteria present in the spleens of representative surviving animals treated with GT-1 or GT-1/GT-055 were tested for susceptibility to GT-1 and the combination, and no differences in MIC compared to the starting isolates were observed (data not shown), which makes it unlikely that the failure of GT-1 or the combination to clear the infections resulted from development of resistance to these agents.

Efficacy of GT-1 with GT-055 was also assessed after an intranasal (IN) challenge of $110 \mathrm{xLD}_{50}$ of $Y$. pestis. Mice that received the lowest doses of either GT-1 $\left(20 \mathrm{mg} \mathrm{kg}^{-1}\right)$ or GT1/GT-055 (20/30 $\left.\mathrm{mg} \mathrm{kg}^{-1}\right)$ succumbed to the infection by day 7. When mice received GT-1 at either 60 or $200 \mathrm{mg} \mathrm{kg}^{-1}$, they had $90 \%$ survival while the combination of GT-1/GT$05560 / 90 \mathrm{mg} \mathrm{kg}^{-1}$ or $200 / 300 \mathrm{mg} \mathrm{kg}^{-1}$ resulted in $80 \%$ or $100 \%$ survival, respectively (Fig. 2). The bacterial load in the lungs and spleens from 3 mice that survived in each group were determined. The spleens isolated from the surviving mice had no detectable bacteria, but at least one mouse from each group had bacteria present in the lungs (Table 6).

\section{Discussion}

The incorporation of an iron-binding siderophore moiety with an antibiotic is designed to act as a "Trojan horse", delivering 
Table $3 \mathrm{MIC}_{90}$ and range of $\mathrm{MICs}^{\mathrm{a}}$ against biothreat bacterial pathogens

\begin{tabular}{lllll}
\hline Compounds & Media & B. pseudomallei & B. mallei & Y. pestis \\
\hline GT-1 & CAMHB & $\leq 0.03(\leq 0.03-0.5)$ & $32(4-32)$ & $2(0.12-4)$ \\
& ID-CAMHB & $0.12(\leq 0.03-0.12)$ & $8(2-16)$ & $0.5(\leq 0.03-0.5)$ \\
GT-1/GT-055 (2:1) & CAMHB & $\leq 0.03 / \leq 0.015(\leq 0.03 / \leq 0.015-0.25 / 0.125)$ & $8 / 4(2 / 1-16 / 8)$ & $2 / 1(0.12 / 0.06-2 / 1)$ \\
& ID-CAMHB & $0.25 / 0.125(\leq 0.03 / \leq 0.015-8 / 4)$ & $4 / 2(2 / 1-8 / 4)$ & $0.5 / 0.25(0.06 / 0.03-1 / 0.5)$ \\
GT-1 GT-055 $(4 \mu \mathrm{g} / \mathrm{ml})$ & CAMHB & $\leq 0.03 / 4(\leq 0.03 / 4)$ & $8 / 4(1 / 4-16 / 4)$ & $1 / 4(\leq 0.03 / 4-2 / 4)$ \\
& ID-CAMHB & $0.06 / 4(\leq 0.03 / 4-4 / 4)$ & $2 / 4(0.5 / 4-4 / 4)$ & $0.12 / 4(\leq 0.03 / 4-0.25 / 4)$ \\
GT-055 & CAMHB & $>32(>32)$ & $>32(32->32)$ & $>32(8->32)$ \\
& ID-CAMHB & $>32(>32)$ & $>32(>-32)$ & $>32(2->32)$ \\
DOX & CAMHB & $2(0.25-2)$ & $0.12(\leq 0.015-0.25)$ & $1(0.12-1)$ \\
& ID-CAMHB & $1(0.5->32)$ & $0.25(\leq 0.015-0.5)$ & $1(0.12-2)$ \\
\hline
\end{tabular}

${ }^{\mathrm{a}} \mathrm{MIC}(\mu \mathrm{g} / \mathrm{ml})$ of GT-1 or GT-055 or GT-1/GT-055 in combination

Table 4 Summary of GT-1 and GT-1/GT-055 efficacy in mouse model of B. pseudomallei infection

\begin{tabular}{llllll}
\hline Cohort & Antibiotic & Dose & \# Deaths & Median survival (Days post-challenge) & $\%$ Survival \\
\hline 1 & Saline & q6 & 10 & 6 & 0 \\
2 & Ceftazidime & $300 \mathrm{mg} / \mathrm{kg}, \mathrm{q} 6$ & 2 & Undefined & 7.5 \\
3 & GT-1 & $30 \mathrm{mg} / \mathrm{kg}, \mathrm{q} 6$ & 10 & 8.5 & 0 \\
4 & GT-1 & $60 \mathrm{mg} / \mathrm{kg}, \mathrm{q} 6$ & 10 & 8 & 0 \\
5 & GT-1 & $120 \mathrm{mg} / \mathrm{kg}, \mathrm{q} 6$ & 10 & 12 & 10 \\
6 & GT-1 & $120 \mathrm{mg} / \mathrm{kg}, \mathrm{q} 6,6$-hour start & 9 & 10.5 & 10 \\
7 & GT-1/GT-055 & $30 / 30 \mathrm{mg} / \mathrm{kg}, \mathrm{q} 6$ & 9 & 14.5 & 0 \\
8 & GT-1/GT-055 & $60 / 60 \mathrm{mg} / \mathrm{kg}, \mathrm{q} 6$ & 10 & 42.5 & 30 \\
9 & GT-1/GT-055 & $120 / 120 \mathrm{mg} / \mathrm{kg}, \mathrm{q} 6$ & 7 & 42.5 & 10 \\
10 & GT-1/GT-055 & $120 \mathrm{mg} / \mathrm{kg}, \mathrm{q} 6,6$-hour start & 9 & & \\
\hline
\end{tabular}

Table 5 Bacterial load in spleens of B. pseudomallei-infected and treated mice that survived until day 60 post-infection

\begin{tabular}{lll}
\hline Treatment groups & $\begin{array}{l}\text { Spleen } \\
\text { weight }(\mathrm{g})\end{array}$ & $\begin{array}{l}\text { B. pseudomallei } \\
\text { count (CFU/spleen) }\end{array}$ \\
\hline Ceftazidime 300 mg/kg, q6 & 0.089 & 0 \\
& 0.090 & 0 \\
& 0.084 & 0 \\
& 0.088 & 0 \\
& 0.088 & 0 \\
& 0.090 & 0 \\
& 0.092 & 0 \\
GT-1 120 mg/kg, q6, & 0.098 & 0 \\
6-hour start & 0.719 & $1.80 \mathrm{E}+08$ \\
GT-1/GT-055 & & \\
30/30 mg/kg, q6 & 1.165 & $1.34 \mathrm{E}+08$ \\
GT-1/GT-055 & & \\
120/120 mg/kg, q6 & 0.099 & 0 \\
& 0.365 & 0 \\
GT-1/GT-055 120/120 mg/kg, & 1.889 & 0 \\
q6, 6-hour start & & \\
\hline
\end{tabular}

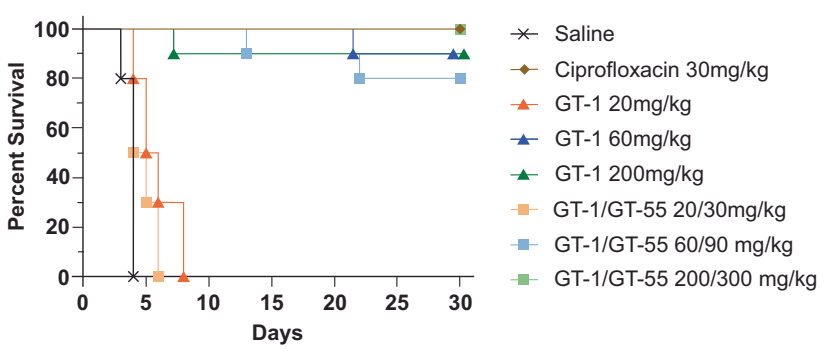

Fig. 2 Efficacy of GT-1 and GT-1/GT-055 in Y. pestis challenge study. Mice infected with $110 \mathrm{X} \mathrm{LD}_{50}$ of $Y$. pestis strain CO92. Treatment with saline (negative control), ciprofloxacin $\left(30 \mathrm{mg} \mathrm{kg}^{-1}, \mathrm{q} 12 \mathrm{~h}\right.$ ), and GT-1 (q6h) or GT-1/GT-55 (q6h) at one of the three specified doses was initiated at $6 \mathrm{~h}$ post-infection and continued for 7 days $(n=10 /$ group). Survival was monitored for 30 days

an antibiotic to a bacterial pathogen via the siderophore-uptake machinery that many bacteria possess in order to obtain the iron needed for survival. This strategy, when incorporated with a novel antibiotic, provides another tool and potential solution to the problem of AMR in specific pathogens. Cefiderocol (formerly S-649266), a siderophore cephalosporin, uses this approach to penetrate through the outer membrane of 
Table 6 Bacterial load in $Y$. pestis mice survivors

\begin{tabular}{lllll}
\hline Treatment & $\begin{array}{l}\text { Spleen } \\
\text { weight }(\mathrm{g})\end{array}$ & $\begin{array}{l}\text { CFU/ } \\
\mathrm{g} \text { spleen }\end{array}$ & $\begin{array}{l}\text { Lung } \\
\text { weight }(\mathrm{g})\end{array}$ & $\begin{array}{l}\text { CFU/g } \\
\text { lung } \\
\left(\times 10^{3}\right)\end{array}$ \\
\hline Ciprofloxacin 30 & 0.100 & 0 & 0.232 & 0 \\
mg/kg & 0.107 & 0 & 0.313 & 16.0 \\
& 0.097 & 0 & 0.401 & 12.5 \\
GT-1 60 mg/kg & 0.103 & 0 & 0.383 & 13.1 \\
& 0.192 & 0 & 0.256 & 19.5 \\
GT-1 200 mg/kg & 0.086 & 0 & 0.356 & 0 \\
& 0.107 & 0 & 0.526 & 0.5 \\
GT-1/GT-055 & 0.078 & 0 & 0.315 & 1.1 \\
60/90 mg/kg & 0.093 & 0 & 0.381 & 13.1 \\
& 0.087 & 0 & 0.237 & 21.1 \\
GT-1/GT-055 & 0.103 & 0 & 0.313 & 0 \\
200/300 mg/kg & 0.087 & 0 & 0.267 & 18.7 \\
& 0.073 & 0 & 0.332 & 0 \\
& 0.072 & 0 & 0.226 & 0 \\
\hline
\end{tabular}

Gram negative bacteria and thereby gain access to the penicillin binding protein targets of inhibition and is approved for use without a $\beta$-lactamase inhibitor [9].

GT-1 contains a siderophore fused to a novel cephalosporin to create such a molecule. Of the panel of nosocomial and community-acquired bacterial pathogens tested, the combination of GT-1 and a broad-spectrum BLI, GT-055, demonstrated the greatest in vitro efficacy versus $E$. coli and E. cloacae, with $>80 \%$ of strains tested yielding sub- $4 \mu \mathrm{g} \mathrm{ml}{ }^{-1}$ MIC values. Of the 16 bacterial pathogens tested, $68 \%$ had sub- $4 \mu \mathrm{g} \mathrm{ml}^{-1}$ MIC values. These results are consistent with the susceptibility of clinical isolates of the ESKAPE pathogens to GT-1 and GT-055 in previous studies [6, 8]. The presence of NDM-1 seemed to correlate with resistance in the strains tested here, as $23 / 28$ strains with that enzyme were resistant to the combination. A previous study examined the $\beta$-lactamases present in the clinical isolates that were tested for susceptibility to GT-1 and/or GT-055, but NDM-1 was not represented [8]. When cefiderocol was tested against 49 NDM-1-positive E. coli, K. pneumoniae, and other Enterobacterales, only 5 E. coli strains were resistant to the antibiotic [7]. Further characterization of clinical isolates with NDM-1 would be required to determine if its presence would confer resistance to the GT-1/GT-055 combination.

The combination of GT-1 and GT-055 was particularly effective in vitro against the biothreat pathogens B. pseudomallei and $Y$. pestis. Cefiderocol has also demonstrated potent in vitro activity against these biothreat pathogens $[22,23]$, although there are no published studies of its in vivo activity against these pathogens in the mouse models of infection. Our present studies demonstrate that
GT-1 alone or in combination with GT-055 has potent activity against $Y$. pestis in a murine model of intranasal infection. The highest dose of GT-1/GT-055 resulted in $100 \%$ survival of the mice, equivalent to the cohort treated with ciprofloxacin. When mice were examined for bacterial burden in the lungs at the end of study, one of three mice from the GT-1/GT-055 highest dose group had bacteria present, whereas two of three mice treated with ciprofloxacin had bacteria present. The highest doses of GT-1/ GT-055 were comparable to ciprofloxacin in their ability to protect the mice post-infection and could be a feasible treatment options if those doses were achievable in patients.

We initiated treatment of the $Y$. pestis-infected mice at $6 \mathrm{~h}$ post-infection and saw at least $80 \%$ survival in the two highest doses of GT- 1 alone and GT-1/GT-055. The use of $\beta$ lactam antibiotics to treat $Y$. pestis infections is contraindicated. Previous mouse studies observed that mice infected via aerosol exposure to $Y$. pestis that received the $\beta$-lactam antibiotics cephalosporins, ampicillin, or aztreonam, at $42 \mathrm{~h}$ post-infection succumbed to infection faster than the mice that received a saline control [24]. These antibiotics were moderately effective when treatment was initiated at $24 \mathrm{~h}$ postinfection, with survival rates between 25 and $100 \%$ for different cephalosporins but were, for the most part, sub-optimal compared to the $100 \%$ survival observed after ciprofloxacin treatment. A similar decline in efficacy in later treatment initiation was observed in another study that investigated the efficacy of imipenem and ceftazidime as treatments after aerosol exposure to $Y$. pestis [25]. That study also analyzed the cytokine profiles and release of endotoxin after treatment initiation. The release of endotoxin after $\beta$-lactam treatment is a concern, but the ciprofloxacin treated-mice released more endotoxin than was seen after treatment with either ceftazidime or imipenem. The potential effect of ciprofloxacin on other toxins produced by $Y$. pestis was not examined. Treatment with any of the three antibiotics resulted in the release of less endotoxin than was observed in the saline-treated control group [25]. It is unclear if a later initiation time would have also resulted in a similar decline in efficacy of GT-1 or GT-1/ GT-055, but it seems possible.

The combined treatment of GT-1/GT-055 increased the mean time until death in mice infected with $B$. pseudomallei. However, a bacterial load was still evident in the spleen of the few survivors in all but one group, GT-1/GT-055 120/120 mg $\mathrm{kg}^{-1}$. It is not clear why the very potent activity of GT-1 and GT-1/GT-055 against $B$. pseudomallei observed in vitro is not recapitulated in vivo in the mouse model. The doses tested may have failed to achieve the required concentrations of the drugs at the intracellular location of the bacteria, or differences in expression of iron-uptake systems may occur in vitro and in vivo, thus affecting uptake of GT-1 into the periplasmic space of the pathogen [26]. Additional studies would be required to better understand the basis for these 
findings. Given that less than $50 \%$ of mice survived and the continued bacterial presence in the spleen, suggests that GT-1/ GT-055 is unlikely to be a viable treatment option for $B$. pseudomallei infections.

Here, we have demonstrated a broad-spectrum in vitro activity of GT-1 and GT-1/GT-055 combination treatment against MDR bacteria and biodefense pathogens. The in vitro activity did not translate into efficacy against an in vivo infection model of B. pseudomallei. However, both GT-1 alone and the GT-1/GT-055 combination were effective when administered soon after $Y$. pestis infection in mice.

Acknowledgements This study was supported by funds from the United States Defense Threat Reduction Agency (DTRA). The content is solely the responsibility of the authors and does not necessarily represent the official views of the Department of Army and are not necessarily endorsed by the US Army.

Author contributions SAH, JMS, LMP, SL and MML performed the experiments. DB \& BH provided the compounds and advised on dose selection for study design. RGP, SDM, JMS, DB, BH contributed to the design of studies and writing of the mansucript.

\section{Compliance with ethical standards}

Conflict of interest The authors declare no competing interests.

Publisher's note Springer Nature remains neutral with regard to jurisdictional claims in published maps and institutional affiliations.

Open Access This article is licensed under a Creative Commons Attribution 4.0 International License, which permits use, sharing, adaptation, distribution and reproduction in any medium or format, as long as you give appropriate credit to the original author(s) and the source, provide a link to the Creative Commons license, and indicate if changes were made. The images or other third party material in this article are included in the article's Creative Commons license, unless indicated otherwise in a credit line to the material. If material is not included in the article's Creative Commons license and your intended use is not permitted by statutory regulation or exceeds the permitted use, you will need to obtain permission directly from the copyright holder. To view a copy of this license, visit http://creativecommons. org/licenses/by/4.0/.

\section{References}

1. CDC. Antibiotic resistance threats in the United States, 2019 (2019 AR Threats Report). In: Services USDoHaH, editor. Atlanta, GA: CDC; 2019.

2. Rice LB. Federal funding for the study of antimicrobial resistance in nosocomial pathogens: no ESKAPE. J Infect Dis. 2008;197:1079-81.

3. Boucher HW, Talbot GH, Bradley JS, Edwards JE, Gilbert D, Rice LB, et al. Bad bugs, no drugs: no ESKAPE! An update from the Infectious Diseases Society of America. Clin Infect Dis. 2009;48:1-12.

4. Rhodes KA, Schweizer HP. Antibiotic resistance in Burkholderia species. Drug Resist Updat. 2016;28:82-90.

5. Dennis DT, Gage KL, Gratz NG, Poland JD, Tikhomirov E, World Health Organization. Epidemic Disease Center. Plague manual: epidemiology, distribution, surveillance and control/ principal authors: David T Dennis. [et al.]. Geneva: World Health Organization; 1999.

6. Oh SH, Park HS, Kim HS, Yun JY, Oh K, Cho YL, et al. Antimicrobial activities of LCB10-0200, a novel siderophore cephalosporin, against the clinical isolates of Pseudomonas aeruginosa and other pathogens. Int $\mathrm{J}$ Antimicrob Agents. 2017;50:700-6.

7. Kohira N, West J, Ito A, Ito-Horiyama T, Nakamura R, Sato T, et al. In vitro antimicrobial activity of a siderophore cephalosporin, S-649266, against enterobacteriaceae clinical isolates, including carbapenem-resistant strains. Antimicrob Agents Chemother. 2016; 60:729-34.

8. Nguyen LP, Pinto NA, Vu TN, Lee H, Cho YL, Byun JH, et al. In vitro activity of a novel siderophore-cephalosporin, GT-1 and serine-type beta-lactamase inhibitor, GT-055, against Escherichia coli, Klebsiella pneumoniae and Acinetobacter spp. panel strains. Antibiotics. 2020;9. https://doi.org/10.3390/antibiotics9050267.

9. Abdul-Mutakabbir JC, Alosaimy S, Morrisette T, Kebriaei R, Rybak MJ. Cefiderocol: a novel siderophore cephalosporin against multidrug-resistant gram-negative pathogens. Pharmacotherapy. 2020;40:1228-47.

10. Mushtaq S, Sadouki Z, Vickers A, Livermore DM, Woodford N. In vitro activity of cefiderocol, a siderophore cephalosporin, against multidrug-resistant gram-negative bacteria. Antimicrob Agents Chemother. 2020;64;e01582-20.

11. Kwon HJCYL, Hannah B. In vitro ADME and in vivo pharmacokinetic profiles of GT-1, a novel siderophore cephalosporin, in the mouse, rat, and dog. American Society for Microbiology Microbe; June 7-11; Atlanta, GA, 2018.

12. Lakota EAL, Zhao AM, Thye D, VanScoy BD, Bhavnani SM, Ambrose PG, et al. Pharmacokinetic-pharmacodynamic (PK-PD) analyses for LCB10-0200 (GT-1) using data from a murine-thigh infection model. American Association of Microbiology Microbe; June 1-5; New Orleans, LA, 2017.

13. Lakota EALA, Zhao M, Bader C, Taylor DS, Biek D, Cho YL, et al. Pharmacokinetic-pharmacodynamic targets for GT-1 efficacy using a murine-lung infection model. American Society for Microbiology Microbe; June 7-11; Atlanta, GA, 2018.

14. VanScoy BDTM, Conde1 H, Bhavnani SM, Biek D, Hannah B, Thye D, et al. Pharmacokinetics-pharmacodynamics of the novel beta-lactamase inhibitor GT-055 in combination with the siderophore cephalosporin, GT-1. In: Proceedings of the 29th European congress of clinical microbiology and infectious diseases; April 13-16; Amsterdam, NL, 2019.

15. CDC. AR Isolate Bank 2021. https://wwwn.cdc.gov/ARIsolateBank/.

16. CLSI. Methods for dilution antimicrobial susceptibility tests for bacteria that grow aerobically, 11th ed. Clinical and Laboratory Standards Institute, Wayne, PA. 2018.

17. CLSI. Performance standards for antimicrobial susceptibility testing, 29th ed. Clinical and Laboratory Standards Institute, Wayne, PA. 2019.

18. May KR. The collison nebulizer: description, performance and application. J Aerosol Sci. 1973;4:235-43.

19. Hartings JM, Roy CJ. The automated bioaerosol exposure system: preclinical platform development and a respiratory dosimetry application with nonhuman primates. J Pharm Toxicol Methods. 2004;49:39-55.

20. Guyton AC. Measurement of the respiratory volumes of laboratory animals. Am J Physiol. 1947;150:70-7.

21. Lathem WW, Crosby SD, Miller VL, Goldman WE. Progression of primary pneumonic plague: a mouse model of infection, pathology, and bacterial transcriptional activity. Proc Natl Acad Sci USA. 2005;102:17786-91.

22. Tsuji M, Jakielaszek C, Marchand LCL. S-649266, a novel siderophore cephalosporin: in vitro activity against biothreat pathogen. Open Forum Infect Dis. 2016;3;S515. 
23. Burnard D, Robertson G, Henderson A, Falconer C, Bauer MJ, Cottrell $\mathrm{K}$, et al. Burkholderia pseudomallei clinical isolates are highly susceptible in vitro to cefiderocol, a siderophore cephalosporin. Antimicrob Agents Chemother. 2021;65;e00685-20.

24. Byrne WR, Welkos SL, Pitt ML, Davis KJ, Brueckner RP, Ezzell JW, et al. Antibiotic treatment of experimental pneumonic plague in mice. Antimicrob Agents Chemother. 1998;42:675-81.
25. Heine HS, Louie A, Adamovicz JJ, Amemiya K, Fast RL, Miller L, et al. Evaluation of imipenem for prophylaxis and therapy of Yersinia pestis delivered by aerosol in a mouse model of pneumonic plague. Antimicrob Agents Chemother. 2014;58:3276-84.

26. Page MGP. The role of iron and siderophores in infection, and the development of siderophore antibiotics. Clin Infect Dis. 2019;69: S529-S37. Suppl 7. 